\title{
Dirichlet boundary conditions in a noncommutative theory
}

\author{
C. D. Fosco and P. Scuracchio \\ Centro Atómico Bariloche and Instituto Balseiro \\ Comisión Nacional de Energía Atómica \\ R8402AGP Bariloche, Argentina.
}

August 3, 2021

\begin{abstract}
We study the problem of imposing Dirichlet-like boundary conditions along a static spatial curve, in a planar Noncommutative Quantum Field Theory model.

After constructing interaction terms that impose the boundary conditions, we discuss their implementation at the level of an interacting theory, with a focus on their physical consequences, and the symmetries they preserve. We also derive the effect they have on certain observables, like the Casimir energies.
\end{abstract}




\section{Introduction}

Casimir and related effects [1] may be thought of as observable results of the interplay between the geometrical properties of a certain spatial region $\mathcal{M}$, subject to some conditions imposed on its boundary $\partial \mathcal{M}$, and the vacuum fluctuations (inside and outside $\mathcal{M}$ ) of a quantum field that experiences those boundary conditions. One of the best-known observable consequences of this interplay is the Casimir force, whose properties depend on the details of the system considered, like the nature of the boundary conditions to be imposed, the number of spatial dimensions, and the kinematic properties of the vacuum field itself (like its spin and internal space structure).

A very special case occurs when the vacuum field is described by a Noncommutative Quantum Field Theory (NCQFT) [2], since this single quality simultaneously affects all of the properties which observable effects may depend upon. Indeed, a first, immediate fact, is that the spacetime structure where one should define $\mathcal{M}$ and $\partial \mathcal{M}$, is different to the one of a commutative theory, since the coordinates $x_{\mu}$ are noncommutative objects. In this respect, the most frequently considered situation corresponds to assuming that, in $d+1$ dimensions, the coordinates satisfy a relation that falls under the general form:

$$
\left[x_{\mu}, x_{\nu}\right]=i \theta_{\mu \nu}, \quad \mu, \nu=0,1, \ldots, d
$$

where $\theta_{\mu \nu}$ is a constant antisymmetric tensor. At the same time, the field is now an element in the noncommutative algebra generated by the coordinates; thus, one should expect departures from the commutative case also coming from this 'kinematic' aspect.

It is our purpose in this paper to explore some of the distinctive characteristics of this kind of system, namely, NCQFT with static, Dirichlet-like boundary conditions. A very interesting phenomenological approach to this kind of problem has been applied, in [3, to the Casimir effect for two parallel plates in $3+1$ dimensions. We follow here a different path, where the boundary region appears from the very beginning, and the (unavoidable) 'fuzziness' in the boundaries is automatically incorporated (because of the noncommutativity) into the formulation. Besides, we attempt to make sense of a boundary with a rather arbitrary shape. In this aspect, we generalize the work presented by one of us in [4].

We shall first deal with the formulation of the problem itself, since, as we just mentioned, one needs to make sense of the noncommutative analogue of geometrical regions, for example, $\partial \mathcal{M}$, something that becomes problematic, because of the 'uncertainty principle' which follows from (1). 
An important ingredient in this study is the number of spacetime dimensions of the system considered. We are dealing with static boundaries; with this in mind, we shall study theories where just the spatial coordinates are noncommutative. As a bonus, we avoid muddling the discussion with problems due to time-like noncommutativity, which, although interesting, are not relevant to the kind of phenomenon we want to study here. With this caveat, and restricting our scope to $d \leq 3$, the most interesting case certainly corresponds to $d=2$, namely, planar theories. Indeed, if $d=1$, the theory would be commutative and thus irrelevant to our study. On the other hand, when $d=3$, only two spatial coordinates can be noncommutative. Indeed, in the equation satisfied by the spatial coordinates,

$$
\left[x_{j}, x_{k}\right]=i \theta_{j k}, \quad j, k=1, \ldots, d,
$$

the antisymmetric tensor, being of odd order, has at least a zero eigenvalue; i.e., there is a commutative coordinate. Thus, to make the geometry fully sensitive to noncommutativity, we shall consider a $2+1$ dimensional model.

Finally, there is also a phenomenological reason for this choice: this situation is realized, in exactly that way, when a strong constant magnetic field is applied to an essentially two-dimensional system. A projection to the lowest Landau level then justifies a noncommutative description [5, 6]. In this kind of planar model, boundaries are known to play an important role, and one should indeed expect it to be so because those systems are the quantum version of incompressible fluids [7, 8].

From a practical standpoint, since the time coordinate remains commutative, the Hamiltonian plays the role of generator of infinitesimal time translations in the usual way, hence many standard Quantum Field Theory tools retain the usual interpretation they have in the commutative case.

The structure of this paper is as follows: in section 2 we define the model and introduce our notation and conventions, in section 3 we study the boundary conditions and discuss their main properties. In 4, we deal with the case of models including a Grosse-Wulkenhaar term, at the self-dual point. Section 5 contains our conclusions.

\section{The model}

We shall be concerned with a complex noncommutative scalar field $\phi$ in $2+1$ dimensions, on which we shall define and impose (the noncommutative version of) Dirichlet-like boundary conditions along a spatial curve $\mathcal{C}$ (see Figure 1), defined, for example, by its parametric form:

$$
\text { C) } \xi \longrightarrow z(\xi)
$$




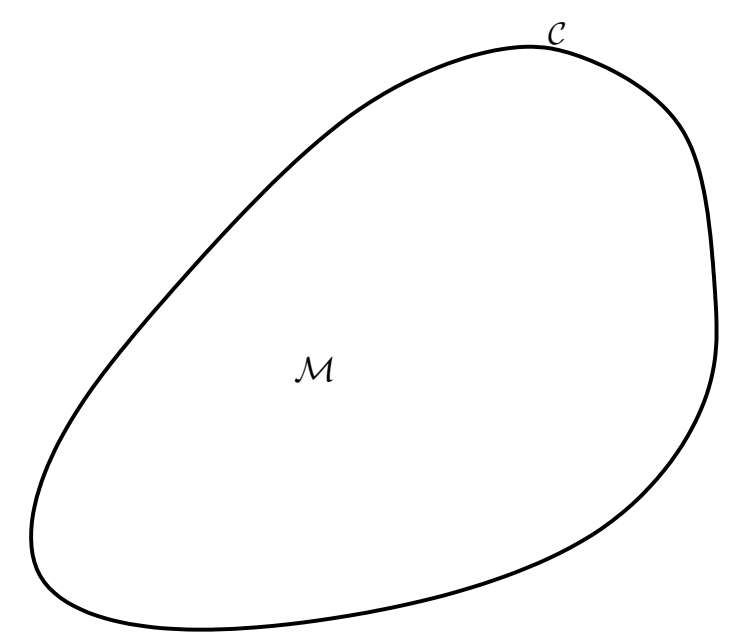

Figure 1: The region $\mathcal{M}$ and its boundary $\mathcal{C}$

where $\xi$ is a real parameter. We shall use, throughout this paper, the convention that spatial coordinates shall be denoted by letters from the end of the Roman alphabet $(x, y, z, \ldots)$, so that the noncommutativity relations (which, by assumption, affect just those coordinates) may be written as follows:

$$
\left[x_{j}, x_{k}\right]=i \theta_{j k}, \theta_{j k}=\theta \epsilon_{j k}, \quad j, k=1,2 .
$$

where $\theta$, the parameter controlling the strength of noncommutativity, has the dimensions of an area. The (commutative) Euclidean time coordinate, shall be denoted by $\tau$ or $x_{0}$, depending on the context. The curve $\mathcal{C}$ defined above is a standard, commutative geometry object. To impose boundary conditions on the NCQFT, we follow the approach of [4], where Dirichletlike conditions are imposed by means of a specific interaction term which makes use of the noncommutative version of a $\delta$-like interaction term. This will (as we shall see) induce some special conditions for the fields on a 'fuzzy' region determined by the curve, at the same time preserving the property of having the right commutative limit.

The scalar field $\phi$ will be equipped with an Euclidean action $S$, a functional of the curve $\mathcal{C}$, the field $\phi$ and its adjoint $\phi^{\dagger}$, with the following structure:

$$
\begin{aligned}
S\left(\mathcal{C} ; \phi^{\dagger}, \phi\right) & =S_{0}\left(\mathcal{C} ; \phi^{\dagger}, \phi\right)+S_{I}\left(\phi^{\dagger}, \phi\right) \\
S_{0}\left(\mathcal{C} ; \phi^{\dagger}, \phi\right) & =S_{f}\left(\phi^{\dagger}, \phi\right)+S_{b}\left(\mathcal{C} ; \phi^{\dagger}, \phi\right)
\end{aligned}
$$


where $S_{f}$ denotes the action for a non-interacting field in the absence of borders, $S_{b}$ is a term which accounts for the boundary conditions, and $S_{I}$ corresponds to self-interactions. More explicitly, we shall assume for $S_{f}$ the form:

$$
S_{f}\left(\phi^{\dagger}, \phi\right)=\int d^{3} x\left[\left(\partial_{\mu} \phi\right)^{\dagger} \star \partial_{\mu} \phi+m^{2} \phi^{\dagger} \star \phi\right]
$$

while for $S_{b}$, inspired by the commutative case, we consider two non-equivalent forms, $S_{b}^{(L)}$ and $S_{b}^{(R)}$ (both of which have the same $\theta \rightarrow 0$ limit):

$$
\begin{aligned}
S_{b}^{(L)}\left(\phi^{\dagger}, \phi\right) & =\lambda_{L} \int d^{3} x \phi(x) \star \delta_{\mathcal{C}}(x) \star \phi^{\dagger}(x) \\
S_{b}^{(R)}\left(\phi^{\dagger}, \phi\right) & =\lambda_{R} \int d^{3} x \phi^{\dagger}(x) \star \delta_{\mathcal{C}}(x) \star \phi(x)
\end{aligned}
$$

where $\delta_{\mathcal{C}}(x)$ is the noncommutative analogue of the commutative two-dimensional $\delta_{\mathcal{C}}$ function which has support on $\mathcal{C}$. The effect of adding each one of the boundary terms above plays, shall be elucidated at the end of next section.

In terms of the parametric form for $\mathcal{C}$, the Weyl mapping immediately yields:

$$
\delta_{\mathcal{C}}(x)=\int d \xi|\dot{z}(\xi)| \delta^{(2)}[x-z(\xi)]
$$

with $\dot{z}(\xi) \equiv \frac{d}{d \xi} z(\xi)$. Note that, in the expression above (only) $x_{1}$ and $x_{2}$ are noncommutative objects. It is worth noting that, to make sense of $\delta^{(2)}$ above one makes the implicit assumption that:

$$
\delta^{(2)}[x-z(\xi)]=\int \frac{d^{2} k}{(2 \pi)^{2}} e^{i k \cdot[x-z(\xi)]} .
$$

It will in some cases be useful to consider an alternative implicit representation of the curve. Indeed, assuming that $\mathcal{C}$ can also be described by the equation $\mathcal{F}_{\mathcal{C}}(x)=0$, the commutative version of $\delta_{\mathcal{C}}(x)$ can be written as follows:

$$
\delta_{\mathcal{C}}(x)=\left|\nabla \mathcal{F}_{\mathcal{C}}(x)\right| \delta\left[\mathcal{F}_{\mathcal{C}}(x)\right] .
$$

This representation, except for some special cases, is not suitable for immediate translation to the noncommutative case, because of the possible noncommutativity between the two factors in 10 . Special cases where that problem does not show up are: 1) A linear function, namely: $\mathcal{F}_{\mathcal{C}}=a_{1} x_{1}+a_{2} x_{2}$, which corresponds to a straight line, and $\mathcal{F}_{\mathcal{C}}=\sqrt{x_{1}^{2}+x_{2}^{2}}-R$, which yields a radius $R$ circle. In the general case, $\left|\nabla \mathcal{F}_{\mathcal{C}}\right|$ may fail to commute with $\mathcal{F}_{\mathcal{C}}$. However, except when the gradient vanishes on points belonging to $\mathcal{C}$, one can in principle find an alternative function, $\mathcal{F}_{\mathcal{C}}^{\prime}$ say, such that its gradient does 
have unit modulus on $(\mathcal{C})$, yet defining the same curve. Indeed, one may just define:

$$
\mathcal{F}_{\mathcal{C}}^{\prime}(x)=g(x) \mathcal{F}_{\mathcal{C}}(x)
$$

where $g$ is a function such that:

$$
g(x) \equiv\left|\nabla \mathcal{F}_{\mathcal{C}}(x)\right|^{-1}
$$

for every $x \in \mathcal{C}$. When $x \notin \mathcal{C}, g$ is smoothly extended quite arbitrarily, except for the condition of being positive.

Thus, whenever we use the implicit representation, we shall assume that it has been already 'normalized', such that:

$$
\delta_{\mathcal{C}}(x)=\delta\left[\mathcal{F}_{\mathcal{C}}(x)\right]
$$

which can be unambiguously translated to its noncommutative version 1 . The implementation and meaning of the $S_{b}$ terms are discussed at length in the next section.

Finally, regarding the interaction term, we shall assume the two inequivalent possible ones that can be built out a (globally invariant under $U(1)$ transformations) quartic monomial in the field and its adjoint:

$$
S_{I}=S_{I}^{(1)}+S_{I}^{(2)}
$$

where

$$
\begin{aligned}
S_{I}^{(1)} & =g^{(1)} \int d \tau d^{2} x\left[\phi^{\dagger}(\tau, x) \star \phi(\tau, x) \star \phi^{\dagger}(\tau, x) \star \phi(\tau, x)\right] \\
S_{I}^{(2)} & =g^{(2)} \int d \tau d^{2} x\left[\phi^{\dagger}(\tau, x) \star \phi^{\dagger}(\tau, x) \star \phi(\tau, x) \star \phi(\tau, x)\right],
\end{aligned}
$$

which correspond, respectively, to planar and nonplanar vertices in a perturbative expansion.

We shall make use of the alternative 'operatorial' representation for noncommutative actions, where the fields are not expanded apriori in any basis, since it is useful to prove some special properties. In that representation one has, for example:

$$
\int d^{2} x \phi^{\dagger}(x) \phi(x) \phi^{\dagger}(x) \phi(x) \leftrightarrow 2 \pi \theta \operatorname{Tr}\left[\phi^{\dagger} \phi \phi^{\dagger} \phi\right],
$$

(where we have adopted, for the sake of simplicity, the convention to use the same symbol for a field in the Moyal representation as in the operatorial version).

We conclude this section by noting that except for the boundary terms the action is invariant under the discrete transformation $\phi \rightarrow \phi^{\dagger}, \phi^{\dagger} \rightarrow \phi$, which amounts to a kind of charge conjugation symmetry.

\footnotetext{
${ }^{1}$ As usual, we map functions to Weyl-ordered operators.
} 


\section{The nature of the boundary conditions}

Some general properties can be deduced from the inclusion of the boundary interaction term, $S_{b}$, as defined by a 'normalized' function $\mathcal{F}_{\mathcal{C}}$. It is quite clear that this term, in the commutative limit, yields Dirichlet-like boundary conditions. It is our purpose here to see its effect in the noncommutative case. The intuitive idea that noncommutativity introduces some 'fuzziness' into the game, and therefore the boundary conditions will affect the field on a finite-width region can be made more concrete. Indeed, taking into account the relation exploited in [9], we see that:

$$
S_{b}^{(R)}=\lambda_{R} \int d \tau d^{2} x \phi^{\dagger}(\tau, x) \delta_{\mathcal{C}}\left(x+i \frac{\theta}{2} \tilde{\partial}\right) \phi(x)
$$

with: $\tilde{\partial}_{j} \equiv \epsilon_{j k} \partial_{k}$. Then, by expanding in powers of $\theta: S_{b}^{(R)}=\left.S_{b}^{(R)}\right|_{\theta=0}+$ $\delta S_{b}^{(R)}$, where the first term contains the purely commutative part, and:

$$
\begin{aligned}
\delta S_{b}^{(R)} & =-\frac{i \lambda_{R} \theta}{2} \int d \tau d^{2} x \delta_{\mathcal{C}}(x) \epsilon_{j k} \partial_{j} \phi^{\dagger}(\tau, x) \partial_{k} \phi(\tau, x) \\
& +\frac{\lambda_{R} \theta^{2}}{8} \int d \tau d^{2} x \delta_{\mathcal{C}}(x) \partial_{j_{1}} \partial_{j_{2}} \phi^{\dagger}(\tau, x)\left(\delta_{j_{1} j_{2}} \partial^{2}-\partial_{j_{1}} \partial_{j_{2}}\right) \phi(\tau, x) \\
& +\ldots
\end{aligned}
$$

This means that, at least in an expansion in $\theta$, the boundary condition shall involve not just the field value on the curve but also its values on points that are close to it. This will manifest in the existence of an effective width.

To understand the effect of this term, it is convenient to go back, momentarily, to the operatorial representation of the action. In the $S_{b}^{(R)}$ case, we have:

$$
S_{b}^{(R)}=2 \pi \theta \lambda_{R} \int d \tau \operatorname{Tr}\left[\phi^{\dagger}(\tau, x) \delta_{\mathcal{C}}(x) \phi(\tau, x)\right],
$$

where $\delta_{\mathcal{C}}(x)=\delta\left[\mathcal{F}_{\mathcal{C}}(x)\right]$, and a similar expression for $S_{b}^{(L)}$.

To proceed, it is convenient to introduce a suitable representation (a basis), for the fields, such that the $\delta$-function becomes diagonal. Of course, that is tantamount to $\mathcal{F}_{\mathcal{C}}$ (a self-adjoint operator) itself being diagonal. There is a qualitative difference between two possible cases, according to the spectrum of that function being continuous or discrete. The reason is intimately linked to a geometrical property of the (commutative) curve $\mathcal{C}$ : this curve is assumed to divide space into two ('inner' and 'outer') components: an $\mathcal{F}_{\mathcal{C}}$ with a continuous spectrum corresponds, in the commutative limit, to a case where the two regions are unbounded. A discrete one, on the other hand, amounts to cases where one of the regions has finite area. 
Indeed, the commutative limit may be obtained by assuming that $\theta$ is very small in comparison with the area element. Thus, regarding $\mathcal{F}_{\mathcal{C}}$ as the 'Hamiltonian' for a fictitious time evolution, one can obtain its eigenvalues in a Bohr-Sommerfeld like fashion. Then the area becomes quantized (discrete spectrum) only for a curve that encircles a finite area. Note that this remark yields a possible concrete way to construct the boundary conditions for a given curve: look for a (one dimensional) quantum mechanical Hamiltonian such that, in the semiclassical limit, has a constant energy curve that coincides with the boundary one wants to consider. That Hamiltonian, minus the corresponding energy, is essentially equal to $\mathcal{F}_{\mathcal{C}}$. Of course, one can always use the parametric representation instead.

Simple examples of the two kinds of spectra are: $\mathcal{F}_{\mathcal{C}}=a_{1} x_{1}+a_{2} x_{2}$ (continuous spectrum) and $\mathcal{F}_{\mathcal{C}}=\sqrt{x_{1}^{2}+x_{2}^{2}}-R$ (discrete spectrum). We shall discuss the effect of $S_{b}$ on the properties of the corresponding model for those two cases separately below.

\subsection{Continuous spectrum}

Let $\{|\epsilon\rangle\}$ be an orthonormal basis for $\mathcal{F}_{\mathcal{C}}$ :

$$
\mathcal{F}_{\mathcal{C}}|\epsilon\rangle=\epsilon|\epsilon\rangle, \quad\left\langle\epsilon^{\prime} \mid \epsilon\right\rangle=\delta\left(\epsilon^{\prime}-\epsilon\right) .
$$

We then expand the field in this basis. Using a shorthand notation for the integrals, the expansion amounts to:

$$
\phi(\tau)=\int_{\epsilon, \epsilon^{\prime}} \phi_{\epsilon, \epsilon^{\prime}}(\tau)|\epsilon\rangle\left\langle\epsilon^{\prime}\left|, \quad \phi^{\dagger}(\tau)=\int_{\epsilon, \epsilon^{\prime}} \phi_{\epsilon^{\prime}, \epsilon}^{*}(\tau)\right| \epsilon\right\rangle\left\langle\epsilon^{\prime}\right| .
$$

We then get for $S_{b}^{(R)}$ the following expression:

$$
S_{b}^{(R)}=2 \pi \theta \lambda_{R} \int d \tau \int_{\epsilon}\left|\phi_{0, \epsilon}(\tau)\right|^{2}
$$

which, in the Dirichlet $(\lambda \rightarrow \infty)$ limit imposes the condition:

$$
\phi_{0, \epsilon}(\tau)=\langle 0|\phi(\tau)| \epsilon\rangle=0, \quad \forall \epsilon
$$

or, in terms of operators, $\langle 0| \phi(\tau)=0$ (and $\phi^{\dagger}(\tau)|0\rangle=0{ }^{2}$ ).

\footnotetext{
${ }^{2}$ We have adopted a bra-ket like notation to denote matrix elements of objects in the algebra. Those should not be confused with Fock-space matrix elements of quantum field operators.
} 
On the other hand, an analogous procedure for the $S_{b}^{(L)}$ term, yields:

$$
S_{b}^{(L)}=2 \pi \theta \lambda_{L} \int d \tau \int_{\epsilon}\left|\phi_{\epsilon, 0}(\tau)\right|^{2}
$$

And, in the Dirichlet limit:

$$
\phi_{\epsilon, 0}(\tau)=\langle\epsilon|\phi(\tau)| 0\rangle=0, \quad \forall \epsilon
$$

or, in terms of operators, $\phi(\tau)|0\rangle=0$ (and $\left.\langle 0| \phi^{\dagger}(\tau)=0\right)$.

The two boundary terms can also be written as,

$$
\begin{aligned}
S_{b}^{(R)} & =2 \pi \theta \lambda_{R} \int d \tau \operatorname{Tr}\left[\phi^{\dagger}(\tau) \mathcal{P}_{\mathcal{C}} \phi(\tau)\right] \\
S_{b}^{(L)} & =2 \pi \theta \lambda_{L} \int d \tau \operatorname{Tr}\left[\phi(\tau) \mathcal{P}_{\mathcal{C}} \phi^{\dagger}(\tau)\right],
\end{aligned}
$$

with $\mathcal{P}_{\mathcal{C}} \equiv|0\rangle\langle 0|$

To see this kind of constraint at work, we consider a concrete example of a continuous spectrum, or, as already explained, of an unbounded, open curve. The simplest possible case is a straight line: $\mathcal{C} \equiv\left\{\left(x_{1}, x_{2}\right): x_{2}=0\right\}$.

For the sake of concreteness, we consider the case of $S_{b}^{(R)}$, since $S_{b}^{(L)}$ may be obtained by performing quite straightforward changes which we consider at the end of this section (see below).

Rather than using the operator formulation, we go directly to the Moyal representation, to see how it affects the plane-wave solutions one could obtain from considering just $S_{f}$. In this situation, we have:

$$
S_{b}^{(R)}\left(\phi^{\dagger}, \phi\right)=\lambda_{R} \int d \tau \int d^{2} x \phi^{\dagger}(\tau, x) \star \delta\left(x_{2}\right) \star \phi(\tau, x),
$$

which, by Fourier transforming with respect to the translation invariant coordinates, $\tau$ and $x_{1}$, adopts the form:

$$
S_{b}^{(R)}=\lambda_{R} \int \frac{d k_{0}}{2 \pi} \frac{d k_{1}}{2 \pi} \widetilde{\phi}^{*}\left(k_{0}, k_{1} ;-\frac{\theta k_{1}}{2}\right) \widetilde{\phi}\left(k_{0}, k_{1} ;-\frac{\theta k_{1}}{2}\right) .
$$

Thus, we find out the equations of motion that follow from considering just the action defined by $S_{0}$, by performing the same Fourier transformation on the $S_{f}$ term. In real-time:

$$
\left(\partial_{2}^{2}+k_{2}^{2}\right) \widetilde{\phi}\left(k ; x_{2}\right)=\int d x_{2}^{\prime} \widetilde{V}\left(k ; x_{2}, x_{2}^{\prime}\right) \widetilde{\phi}\left(k ; x_{2}^{\prime}\right)
$$


where we have introduced the kernel:

$$
\widetilde{V}\left(k ; x_{2}, x_{2}^{\prime}\right)=\lambda \delta\left(x_{2}+\frac{\theta k_{1}}{2}\right) \delta\left(x_{2}^{\prime}+\frac{\theta k_{1}}{2}\right),
$$

and the mass-shell condition yields: $k_{2} \equiv \sqrt{k_{0}^{2}-k_{1}^{2}}>0$, where we use the positive square-root, to consider a solution which corresponds to a plane wave incident from negative values of $x_{2}$.

This kind of solution is naturally treated as an scattering problem; hence we may write the solution to this problem by means of the corresponding Lippmann-Schwinger (L-S) integral equation:

$$
\begin{aligned}
\widetilde{\phi}\left(k ; x_{2}\right) & =\widetilde{\phi}^{(0)}\left(k ; x_{2}\right) \\
& +\int d x_{2}^{\prime} \int d x_{2}^{\prime \prime} \Delta_{R}\left(k_{2} ; x_{2}, x_{2}^{\prime}\right) \widetilde{V}\left(k ; x_{2}^{\prime}, x_{2}^{\prime \prime}\right) \widetilde{\phi}\left(k, x_{2}^{\prime \prime}\right)
\end{aligned}
$$

where $\widetilde{\phi}^{(0)}$ is the (incident) free-particle wave, solution of

$$
\left(\partial_{2}^{2}+k_{2}^{2}\right) \widetilde{\phi}^{(0)}\left(k_{2} ; x_{2}\right)=0 .
$$

and $\Delta_{R}$ is the retarded Green's function.

Since we assume the free-particle solution to correspond to a wave incident from $x_{2}<0, \widetilde{\phi}^{(0)}\left(k_{2} ; x_{2}\right)=e^{i k_{2} x_{2}}$. Besides, the retarded Green's function satisfies:

$$
\left(\partial_{2}^{2}+k_{2}^{2}\right) \Delta_{R}\left(k_{2} ; x_{2}-x_{2}^{\prime}\right)=\delta\left(x_{2}-x_{2}^{\prime}\right),
$$

(with retarded boundary conditions) and may be written more explicitly as follows:

$$
\Delta_{R}\left(k_{2} ; x_{2}-x_{2}^{\prime}\right)=\frac{i}{2 k_{2}} e^{i k_{2}\left|x_{2}-x_{2}^{\prime}\right|}
$$

To solve the L-S equation, we proceed in an entirely analogous way to the one presented in [10], obtaining:

$$
\widetilde{\phi}\left(k ; x_{2}\right)=e^{i k_{2} x_{2}}+r(k) e^{-i \frac{\theta k_{1} k_{2}}{2}} e^{i k_{2}\left|x_{2}+\frac{\theta k_{1}}{2}\right|},
$$

where: $r(k) \equiv-\frac{\frac{i \lambda}{2 k_{2}}}{1+\frac{i \lambda}{2 k_{2}}}$. From the equation above we can extract, by considering the situations where $x_{2}>0$ and $x_{2}<0$, the properties of the transmitted and reflected waves, respectively. In particular, one may study the dependence of the transmission and reflection coefficients on the incident momentum; in this respect, we recall that the wave also has an $x_{1}$ dependence, which has been factored out of the solution (since it is unaffected by the boundary condition). However, there remains a dependence on $k_{1}$ in the behaviour of the $x_{2}$-dependent part. 
For the transmitted wave, $\widetilde{\phi} \equiv \widetilde{\phi}_{>}$, and we have:

$$
\widetilde{\phi}_{>}\left(k ; x_{2}\right)=[1+r(k)] e^{i k_{2} x_{2}},
$$

whenever $x_{2}+\frac{\theta k_{1}}{2}>0$, exactly as in the commutative case. In particular, there is no transmitted wave when $\lambda \rightarrow \infty$ and $x_{2}+\frac{\theta k_{1}}{2}>0$, since $r(k) \rightarrow-1$. Note, however, that there is an important qualitative difference for points such that $x_{2}+\frac{\theta k_{1}}{2}<0$ (and $x_{2}>0$ ). Here we have instead:

$$
\widetilde{\phi}_{>}\left(k ; x_{2}\right)=e^{i k_{2} x_{2}}+r(k) e^{-i \theta k_{1} k_{2}} e^{-i k_{2} x_{2}},
$$

or, in the Dirichlet limit:

$$
\widetilde{\phi}_{>}\left(k ; x_{2}\right)=e^{i k_{2} x_{2}}-e^{-i \theta k_{1} k_{2}} e^{-i k_{2} x_{2}},
$$

which is quite different to the previous case. In particular, the transmitted current is smaller than for $x_{2}+\frac{\theta k_{1}}{2}>0$, to the point of vanishing in the Dirichlet limit. There is a reflected wave, and the system behaves as if there were a reflecting wall at $x_{2}=-\frac{\theta k_{1}}{2}$, which only acts if $k_{1}<0$ (we assume $\theta>0)$.

The behaviour at $x_{2}<0$ is consistent with this picture. Indeed, if $k_{1}<0$ (for the 'wall' to act), we see that

$$
\widetilde{\phi}_{<}\left(k ; x_{2}\right)=e^{i k_{2} x_{2}}+r(k) e^{-i \theta k_{1} k_{2}} e^{-i k_{2} x_{2}},
$$

since, in this case, $x_{2}+\frac{\theta k_{1}}{2}<0$. We see that the outcome for the reflected wave corresponds to the same result as in the commutative case, except that (due to the extra $\theta$-dependent phase) it corresponds to a wall at $x_{2}=$ $-\frac{\theta k_{1}}{2}>0$, which introduces a spatial shift of twice that value, since the wave has to go forward and bounce back. Finally, also for negative values of $x_{2}$, if $0>x_{2}>-\frac{\theta k_{1}}{2}$, which is nonempty for $k_{1}>0$, we have:

$$
\widetilde{\phi}_{<}\left(k ; x_{2}\right)=[1+r(k)] e^{i k_{2} x_{2}},
$$

meaning that there is no reflected component: it only appears when $x_{2}<$ $-\frac{\theta k_{1}}{2}$.

We may summarize all of the above by the statement that if we imagine sending a wave packet with a non vanishing momentum dispersion in the $x_{1}$ direction, the boundary behaves as it had a finite width $\delta x_{2} \sim \theta \delta k_{1}$. Or, by a standard application of the usual uncertainty principle for $\delta x_{1}$ and $\delta x_{2}$ :

$$
\delta x_{1} \delta x_{2} \sim \theta
$$


In spite of this expected effect, note that, for a single wall one can impose Dirichlet-like boundary conditions. The resulting reflection and transmission coefficients coincide with their commutative counterparts, but the wall will have an effective momentum $\left(k_{1}\right)$ dependent position.

As a consistency check on the previous derivation, we note that condition (23) can be translated into the Fourier transformed fields of the Moyal representation as follows:

$$
0=\int \frac{d^{2} k}{(2 \pi)^{2}}\left\langle 0\left|e^{i k \cdot x}\right| x_{2}\right\rangle \widetilde{\phi}(k) \quad, \quad \forall x_{2} ;
$$

or, more explicitly,

$$
\widetilde{\phi}\left(-\frac{x_{2}}{\theta} ; \frac{x_{2}}{2}\right)=0 \quad, \quad \forall x_{2},
$$

(in the same hybrid Fourier representation we used above). This is of course equivalent to:

$$
\widetilde{\phi}\left(k_{1} ;-\frac{\theta k_{1}}{2}\right)=0 \quad, \quad \forall k_{1}
$$

as it should be.

A similar derivation for the $S_{b}^{(L)}$ term:

$$
S_{b}^{(L)}\left(\phi^{\dagger}, \phi\right)=\lambda \int d \tau \int d^{2} x \phi(\tau, x) \star \delta\left(x_{2}\right) \star \phi^{\dagger}(\tau, x),
$$

after the same Fourier transformation yields:

$$
S_{b}^{(L)}=\lambda \int \frac{d k_{0}}{2 \pi} \frac{d k_{1}}{2 \pi} \widetilde{\phi}^{*}\left(k_{0}, k_{1} ; \frac{\theta k_{1}}{2}\right) \widetilde{\phi}\left(k_{0}, k_{1} ; \frac{\theta k_{1}}{2}\right)
$$

which imposes, in the Dirichlet limit, the condition: $\widetilde{\phi}\left(k_{0}, k_{1} ; \frac{\theta k_{1}}{2}\right)=0, \forall k_{1}$.

A simple extension of the single straight-line case is that of two parallel straight lines, at $x_{2}=0$ and $x_{2}=l$. Since the boundary has two disjoint components, one can consider different choices regarding the $L$ and $R$ boundary terms on each mirror.

In the $R R$ case, the resulting boundary interaction term becomes:

$$
\begin{aligned}
S_{b}^{(R R)} & =\lambda \int \frac{d k_{0}}{2 \pi} \frac{d k_{1}}{2 \pi}\left[\widetilde{\phi}^{*}\left(k_{0}, k_{1} ;-\frac{\theta k_{1}}{2}\right) \widetilde{\phi}\left(k_{0}, k_{1} ;-\frac{\theta k_{1}}{2}\right)\right. \\
& \left.+\widetilde{\phi}^{*}\left(k_{0}, k_{1} ; l-\frac{\theta k_{1}}{2}\right) \widetilde{\phi}\left(k_{0}, k_{1} ; l-\frac{\theta k_{1}}{2}\right)\right] .
\end{aligned}
$$

In particular, when $\lambda \rightarrow \infty$, we find that $\widetilde{\phi}$ has to satisfy the conditions $\widetilde{\phi}\left(k_{0}, k_{1} ;-\frac{\theta k_{1}}{2}\right)=0$ and $\widetilde{\phi}\left(k_{0}, k_{1} ; l-\frac{\theta k_{1}}{2}\right)=0$. The solution is nontrivial 
if $k_{2}=\frac{n \pi}{l}$, exactly as in the commutative case. Thus the Casimir force coincides, in this case, with its commutative counterpart. The same happens when $L L$ conditions are imposed.

The situation changes, however, if one consideres a situation involving $L$ and $R$ terms. Indeed, using $R$ conditions at $x_{2}=l$ and $L$ conditions at $x_{2}=0$, in the Dirichlet limit we obtain: the conditions $\widetilde{\phi}\left(k_{0}, k_{1} ; l-\frac{\theta k_{1}}{2}\right)=0$ and $\widetilde{\phi}\left(k_{0}, k_{1} ; \frac{\theta k_{1}}{2}\right)=0$. The modes are quantized, but now they satisfy:

$$
k_{2}=\frac{n \pi}{\left|l-\theta k_{1}\right|}, \quad n \in \mathbb{N} .
$$

This is of course quite different from the previous case, since we see that, depending on $k_{1}$, the $k_{2}$ allowed values correspond to an effective size $l-\frac{\theta k_{1}}{2}$. This is again consistent with the interpretation that the mirrors are 'displaced' by a momentum-dependent amount. In the hybrid case, the displacement is symmetrical about $x_{2}=l / 2$, thus it produces an observable effect (unlike what happens in the $R R$ and $L L$ cases). Note that the hybrid case is more symmetrical than those cases, since there is a parity symmetry, in the boundary interaction term, with respect to the middle point $x_{2}=l / 2$.

The Casimir energy per unit length $\mathcal{E}$ (i.e., tension) corresponding to this case is straightforwardly evaluated, the result being:

$$
\mathcal{E}=\frac{1}{2} \int \frac{d k_{0} d k_{1}}{(2 \pi)^{2}} \ln \left[1-e^{-2 \sqrt{k_{0}^{2}+k_{1}^{2}}\left|l-\theta k_{1}\right|}\right]
$$

This tension tends to its proper commutative limit when $l^{2}>>\theta$. However, in the opposite (short distance) limit it grows faster than $l^{-2}$.

We can calculate the free propagator (corresponding to $S_{0}$ ) for the Dirichlet limit:

$$
\begin{aligned}
\Delta\left(k_{\|} ; x_{2}, y_{2}\right) & =\frac{1}{2 k_{\|}}\left[e^{-k_{\|}\left|x_{2}-y_{2}\right|}\right. \\
& -\frac{1}{1-e^{-2 k_{\|}\left|l-\theta k_{1}\right|}} e^{-k_{\|}\left(\left|x_{2}-l+\frac{\theta k_{1}}{2}\right|+\left|y_{2}-l+\frac{\theta k_{1}}{2}\right|\right)} \\
& -\frac{1}{1-e^{-2 k_{\|}\left|l-\theta k_{1}\right|}} e^{-k_{\|}\left(\left|x_{2}-\frac{\theta k_{1}}{2}\right|+\left|y_{2}-\frac{\theta k_{1}}{2}\right|\right)} \\
& +\frac{e^{-k_{\|}\left|l-\theta k_{1}\right|}}{1-e^{-2 k_{\|}\left|l-\theta k_{1}\right|}} e^{-k_{\|}\left(\left|x_{2}-l+\frac{\theta k_{1}}{2}\right|+\left|y_{2}-\frac{\theta k_{1}}{2}\right|\right)} \\
& \left.+\frac{e^{-k_{\|}\left|l-\theta k_{1}\right|}}{1-e^{-2 k_{\|}\left|l-\theta k_{1}\right|}} e^{-k_{\|}\left(\left|x_{2}-\frac{\theta k_{1}}{2}\right|+\left|y_{2}-l+\frac{\theta k_{1}}{2}\right|\right)}\right]
\end{aligned}
$$

Finally, note that there is no impediment to include both kinds of boundary term at the same point. The result of this procedure amounts to, in the 
Dirichlet limit, imposing $\langle 0|\phi| \epsilon\rangle=\langle\epsilon|\phi| 0\rangle=0, \forall \epsilon$. The corresponding propagator, for the case of two mirrors at $x_{2}=0$, may be obtained by setting $l=0$ above:

$$
\begin{aligned}
\Delta\left(k_{\|} ; x_{2}, y_{2}\right) & =\frac{1}{2 k_{\|}}\left[e^{-k_{\|}\left|x_{2}-y_{2}\right|}-e^{-k_{\|}\left(\left|x_{2}+\frac{\theta k_{1}}{2}\right|+\left|y_{2}+\frac{\theta k_{1}}{2}\right|\right)}\right. \\
& \left.-e^{-k_{\|}\left(\left|x_{2}-\frac{\theta k_{1}}{2}\right|+\left|y_{2}-\frac{\theta k_{1}}{2}\right|\right)}\right] .
\end{aligned}
$$

Besides, it is not difficult to see that when a wave packet insides on this kind of mirror, there is an effective 'widening' (depending on $k_{1}$ ) as a result of the emergence momentum dependent boundary condition to the left and right of $x_{2}=0$. Note that, imposing both conditions yields a more symmetrical situation, in the sense that the system is even under parity and under charge conjugation: $\phi \rightarrow \phi^{\dagger}, \phi^{\dagger} \rightarrow \phi$.

If one rederives the allowed spectra for two of these mirrors, one finds again (48), plus a condition on $k_{1}$ :

$$
k_{1}=\frac{l}{\theta} r
$$

where $r$ is an arbitrary rational number.

\subsection{Discrete spectrum}

In this case, we use instead a discrete basis $\{|n\rangle\}$ :

$$
\mathcal{F}_{\mathcal{C}}|n\rangle=\epsilon_{n}|n\rangle, \quad\langle n \mid m\rangle=\delta_{n m} .
$$

Expanding the field in this basis:

$$
\phi(\tau)=\sum_{n m} \phi_{n m}(\tau)|n\rangle\left\langle m\left|, \quad \phi^{\dagger}(\tau)=\sum_{n m} \phi_{m n}^{*}(\tau)\right| n\right\rangle\langle m| .
$$

In order to give meaning to the discrete case, getting a non-vanishing result that moreover is similar to the continuous case, we use, in this case, a Kronecker $\delta$ (rather that a Dirac one) such that:

$$
S_{b}^{(R)}=2 \pi \theta \lambda_{R} \int d \tau \sum_{n}\left|\phi_{0 n}(\tau)\right|^{2}
$$

which in the Dirichlet $\left(\lambda_{R} \rightarrow \infty\right)$ limit imposes the condition

$$
\phi_{0 n}(\tau)=\langle 0|\phi(\tau)| n\rangle=0, \forall n .
$$


So we take this kind of condition as the starting point of our derivation in the discrete spectrum. Note that, in order for the condition on the field to be non-empty, one should make sure that 0 belongs to the spectrum of $\mathcal{F}_{\mathcal{C}}$. Besides, the normalization of the $\delta$ function loses its meaning here since one has a sum rather than integral. It is quite natural to use, as the analogue continuous case, the following:

$$
\delta_{\mathcal{C}}=\mathcal{P}_{\mathcal{C}}
$$

where $\mathcal{P}_{\mathcal{C}}$ is the orthogonal projector on the null space of the operator $\mathcal{F}_{\mathcal{C}}$. In the notation used above: $\mathcal{P}_{\mathcal{C}}=|0\rangle\langle 0|$.

The example we shall produce for this case will be the one of a circle of radious $R$, with $\mathcal{F}_{\mathcal{C}}=\delta\left(\sqrt{x_{1}^{2}+x_{2}^{2}}-R\right)$. Since $\left[x_{1}, x_{2}\right]=i \theta$, one introduces $a=\left(x_{1}+i x_{2}\right) / \sqrt{2 \theta}$ and $a^{\dagger}=\left(x_{1}-i x_{2}\right) / \sqrt{2 \theta}$. Then:

$$
\mathcal{F}_{\mathcal{C}}=\sqrt{2 \theta}\left(\sqrt{n+1 / 2}-\frac{R}{\sqrt{2 \theta}}\right), \quad n=a^{\dagger} a .
$$

Besides, $R$ is chosen in order to ensure 0 belong to the spectrum of $\mathcal{F}_{\mathcal{C}}$. Thus:

$$
\frac{R}{\sqrt{2 \theta}}=\sqrt{N+\frac{1}{2}}, \quad N=0,1, \ldots
$$

As in the continuous case, one can impose both $R$ and $L$ conditions simultaneously.

\subsection{Symmetries}

We have, in the previous cases, expanded the operators in a basis which diagonalizes the $\delta$-function; in other words, a basis which is consistent with the symmetries that survive the imposition of the boundary conditions. A particularly interesting case arises when the same basis also diagonalizes the $S_{f}$ term, since in such a case the free propagator (and, as a by-product, the energies) can be exactly found. However, the only two known cases in which $S_{f}$ can be exactly diagonalized correspond to free fields or to a field in the presence of a 'critical magnetic field', which renders the model selfdual under the Langmann-Szabo duality [11], or to the presence of GrosseWulkenhaar [12] confining potentials. The basis which renders the propagator diagonal is the so-called 'matrix base', and corresponds to harmonic oscillator like states created by $a^{\dagger} \equiv \frac{x_{1}-i x_{2}}{\sqrt{2 \theta}}$. Thus, the only non-trivial case where the two symmetries agree, correspond to the circular boundaries, whose radii are determined by the discrete eigenvalue of the number operator $a^{\dagger} a$. They are considered in the following section. 


\section{Self-dual models}

We have seen that the only system where one can find a basis that simultaneously diagonalizes $S_{f}$ and $S_{b}$ consists of a Langmann-Szabo self-dual action and a circular boundary. To construct self-dual models one can introduce a coupling between $\phi$ and a critical magnetic field, or to use a GrosseWulkenhaar confining potential term. In the former, one uses one of two constant magnetic fields, $B_{L}$ and $B_{R}$, corresponding to fundamental and anti-fundamental $U_{\star}(1)$ gauge transformation properties, respectively. The covariant derivatives act on $\phi$ as follows:

$$
\begin{aligned}
D_{\mu}^{(L)} \phi & =\partial_{\mu} \phi+i A_{\mu}^{(L)} \star \phi \\
D_{\mu}^{(R)} \phi & =\partial_{\mu} \phi+i \phi \star A_{\mu}^{(R)},
\end{aligned}
$$

with $A_{j}^{(L, R)} \equiv-\frac{B^{(L, R)}}{2} \epsilon_{j k} x_{k}$, and $A_{0}^{(L, R)} \equiv 0$. The resulting 'free' action $S_{f}$ corresponding to each case is:

$$
\begin{aligned}
S_{f}^{(R)} & =\int d^{3} x\left[\left(D_{\mu}^{(R)} \phi\right)^{\dagger} \star D_{\mu}^{(R)} \phi+m^{2} \phi^{\dagger} \star \phi\right] \\
S_{f}^{(L)} & =\int d^{3} x\left[\left(D_{\mu}^{(L)} \phi\right)^{\dagger} \star D_{\mu}^{(L)} \phi+m^{2} \phi^{\dagger} \star \phi\right] .
\end{aligned}
$$

Self-duality can be achieved under different conditions, depending on whether $\theta B^{(R)}=-2, \theta B^{(L)}=2$, the resulting free actions being:

$$
\begin{aligned}
S_{f}^{(R)} & =\int d^{3} x \phi^{\dagger} \star\left[-\partial_{\tau}^{2}+\frac{2}{\theta}\left(a^{\dagger} \star a+\frac{1}{2}\right)+m^{2}\right] \star \phi \\
S_{f}^{(L)} & =\int d^{3} x \phi \star\left[-\partial_{\tau}^{2}+\frac{2}{\theta}\left(a^{\dagger} \star a+\frac{1}{2}\right)+m^{2}\right] \star \phi^{\dagger} .
\end{aligned}
$$

The confining potential case, on the other hand, corresponds to an action $S_{f}^{(A)}$ :

$$
\begin{aligned}
S_{f}^{(A)} & =\int d^{3} x\left[\left(\partial_{\mu} \phi\right)^{\dagger} \star \partial_{\mu} \phi+\frac{2}{\theta^{2}} \phi^{\dagger} \star x_{j} \star \phi \star x_{j}+m^{2} \phi^{\dagger} \star \phi\right] \\
& =\int d^{3} x\left\{\phi^{\dagger} \star\left(-\partial_{\tau}^{2}+m^{2}\right) \phi\right. \\
& \left.+\frac{2}{\theta}\left[\phi^{\dagger} \star\left(a^{\dagger} \star a+\frac{1}{2}\right) \phi+\phi \star\left(a^{\dagger} \star a+\frac{1}{2}\right) \phi^{\dagger}\right]\right\} .
\end{aligned}
$$

The most symmetrical action [13] corresponds to the confining potential case, since it has the symmetry under interchange of the field with its adjoint. 
We see here that the there is a natural choice of boundary term: it has to be the sum of $R$ and $L$, with identical coupling constants.

We then write, in the matrix base, the $S_{b}$ action $S_{b}=S_{b}^{(R)}+S_{b}^{(L)}$ for this choice, with $|N\rangle$ denoting the state corresponding to the radius of $\mathcal{C}$ :

$$
\begin{aligned}
S_{b}^{(R)} & =2 \pi \theta \lambda_{R} \int_{\tau} \phi_{N n}^{*}(\tau) \phi_{N n}(\tau) \\
S_{b}^{(L)} & =2 \pi \theta \lambda_{L} \int_{\tau} \phi_{n N}^{*}(\tau) \phi_{n N}(\tau) .
\end{aligned}
$$

The propagator (in frequency space) can be found exactly:

$$
\left\langle\phi_{j k}^{*}(\omega) \phi_{n l}(\omega)\right\rangle=(2 \pi \theta)^{-1} \frac{\delta_{j n} \delta_{k l}}{\omega^{2}+m^{2}+\frac{2}{\theta}(j+k+1)+\lambda\left(\delta_{j N}+\delta_{k N}\right)}
$$

In the Dirichlet limit, one can simply solve the resulting constraints, by restricting the fields to be of the form:

$$
\phi(\tau)=\sum_{n, m \neq N} \phi_{n m}(\tau)|n\rangle\left\langle m\left|, \quad \phi^{\dagger}(\tau)=\sum_{n, m \neq N} \phi_{m n}^{*}(\tau)\right| n\right\rangle\langle m| .
$$

This expansion, when inserted into each one of the interaction terms $S_{I}^{(1,2)}$ may be reinterpreted as having consistently eliminated one of the basis elements, $|N\rangle$.

By the same token, the Casimir energy in this case is just the vacuum energy for the $\phi_{N N}$ field mode. This object has the same action as a single oscillator, thus its vacuum energy is: $E=\sqrt{\frac{2}{\theta}}(2 N+1)$. In terms of $R$, this corresponds to a tension:

$$
\mathcal{E}=\frac{1}{2 \pi} \frac{R}{\theta^{3 / 2}}
$$

\section{Conclusions}

We have studied different ways to impose boundary conditions on a noncommutative QFT. We have seen that, for the case of a complex field, there are in principle two inequivalent terms which, however, have the same commutative limit. Those terms introduce qualitatively different boundary conditions, and respect different symmetries. We have shown that they may be interpreted producing (under certain circumstances) a 'widening' of the curve, such that that effect is compatible with the uncertainty principle for the coordinates. We also considered self-dual models, showing that in the most symmetrical situation, imposing Dirichlet conditions is equivalent to discarding one of 
the elements from the matrix base. Namely, to reducing the space of field configurations. That reduction can be consistently implemented even when there are interactions, something that does not happen in for non-symmetric boundary terms.

\section{Acknowledgements}

C. D. F. acknowledges support from CONICET, CNEA, ANPCyT and UNCuyo (Argentina). P. S. has been supported by a Petroenergy SA - Trafigura studentship at Instituto Balseiro, UNCuyo.

\section{References}

[1] M. Bordag, U. Mohideen, and V. M. Mostepanenko, Phys. Rep. 353, 1 (2001); K. A. Milton, The Casimir Effect: Physical Manifestations of the Zero-Point Energy (World Scientific, Singapore, 2001); S. Reynaud et al., C. R. Acad. Sci. Paris IV-2, 1287 (2001); K. A. Milton, J. Phys. A: Math. Gen. 37, R209 (2004); S.K. Lamoreaux, Rep. Prog. Phys. 68, 201 (2005); M. Bordag, G.L. Klimchitskaya, U. Mohideen, and V. M. Mostepanenko, Advances in the Casimir Effect, Oxford University Press, Oxford, 2009.

[2] See, for example: M. R. Douglas and N. A. Nekrasov, Rev. Mod. Phys. 73, 977 (2001).

[3] R. Casadio, A. Gruppuso, B. Harms and O. Micu, Phys. Rev. D 76, 025016 (2007).

[4] C. D. Fosco and G. A. Moreno, Phys. Lett. B 659, 901 (2008).

[5] G. V. Dunne and R. Jackiw, Nucl. Phys. Proc. Suppl. 33C, 114 (1993).

[6] C. D. Fosco and A. López, J. Phys. A 37, 4123 (2004).

[7] L. Susskind, "The quantum Hall fluid and non-commutative Chern Simons theory," arXiv:hep-th/0101029.

[8] R. Jackiw, V. P. Nair, S. Y. Pi and A. P. Polychronakos, J. Phys. A 37, R327 (2004).

[9] L. Mezincescu, arXiv:hep-th/0007046. 
[10] C. D. Fosco, F. C. Lombardo and F. D. Mazzitelli, arXiv:0912.0886 [hep-th].

[11] E. Langmann and R. J. Szabo, Phys. Lett. B 533, 168 (2002).

[12] H. Grosse and R. Wulkenhaar, JHEP 0312, 019 (2003).

[13] C. D. Fosco and G. A. Moreno, JHEP11 (2007) 046. 